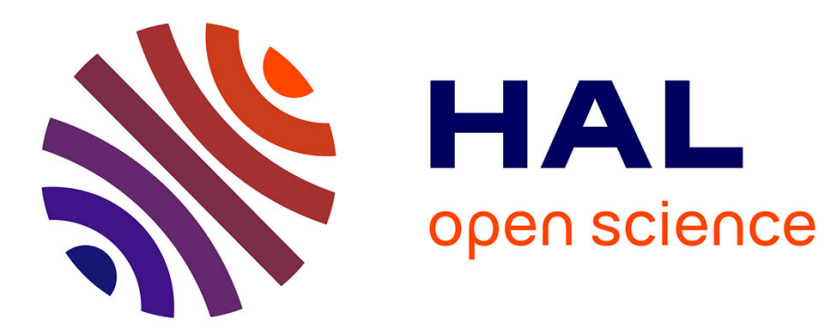

\title{
Stochastic sampling of interaction partners versus deterministic payoff assignment
}

Benno Woelfing, Arne Traulsen

\section{To cite this version:}

Benno Woelfing, Arne Traulsen. Stochastic sampling of interaction partners versus deterministic payoff assignment. Journal of Theoretical Biology, 2009, 257 (4), pp.689. 10.1016/j.jtbi.2008.12.025 . hal-00554555

\section{HAL Id: hal-00554555 \\ https://hal.science/hal-00554555}

Submitted on 11 Jan 2011

HAL is a multi-disciplinary open access archive for the deposit and dissemination of scientific research documents, whether they are published or not. The documents may come from teaching and research institutions in France or abroad, or from public or private research centers.
L'archive ouverte pluridisciplinaire HAL, est destinée au dépôt et à la diffusion de documents scientifiques de niveau recherche, publiés ou non, émanant des établissements d'enseignement et de recherche français ou étrangers, des laboratoires publics ou privés. 


\section{Author's Accepted Manuscript}

Stochastic sampling of interaction partners versus deterministic payoff assignment

Benno Woelfing, Arne Traulsen

PII: $\quad$ S0022-5193(08)00671-1

DOI: $\quad$ doi:10.1016/j.jtbi.2008.12.025

Reference: $\quad$ YJTBI5414

To appear in: $\quad$ Journal of Theoretical Biology

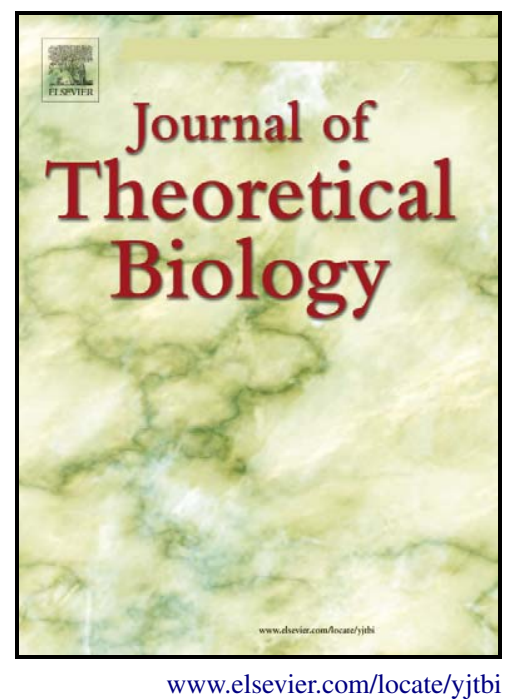

Received date: $\quad 29$ September 2008

Revised date: 18 December 2008

Accepted date: 19 December 2008

Cite this article as: Benno Woelfing and Arne Traulsen, Stochastic sampling of interaction partners versus deterministic payoff assignment, Journal of Theoretical Biology (2008), doi:10.1016/j.jtbi.2008.12.025

This is a PDF file of an unedited manuscript that has been accepted for publication. As a service to our customers we are providing this early version of the manuscript. The manuscript will undergo copyediting, typesetting, and review of the resulting galley proof before it is published in its final citable form. Please note that during the production process errors may be discovered which could affect the content, and all legal disclaimers that apply to the journal pertain. 


\title{
Stochastic sampling of interaction partners versus deterministic payoff assignment
}

\author{
Benno Woelfing and Arne Traulsen \\ Max-Planck-Institute for Evolutionary Biology, August-Thienemann-Str. 2, 24306 Plön, \\ Germany
}

\begin{abstract}
Evolutionary game dynamics describes how successful strategies spread in a population. In well mixed-populations, the usual assumption, e.g. underlying the replicator dynamics, is that individuals obtain a payoff from interactions with a representative sample of the population. This determines their fitness. Here, we analyze a situation in which payoffs are obtained through a single interaction, so that individuals of the same type can have different payoffs. We show analytically that for weak selection, this scenario is identical to the usual approach in which an individual interacts with the whole population. For strong selection, however, differences arise that are reflected in the fixation probabilities and lead to deviating evolutionary dynamics.
\end{abstract}

Key words: Evolutionary game theory, frequency dependent selection, payoff assignment, deterministic versus stochastic payoffs, evolutionary dynamics

\section{Introduction}

If individual fitness depends on the relative abundances of the different phenotypes in the population, frequency-dependent selection is acting. In nature, the outcome of interactions affecting fitness usually depends on the types of individuals involved, so that frequency-dependent selection is common. Prominent examples include assortative mating, reduced viability of mixed pair offspring, self- 
incompatibility alleles in plants and competition for resources with neighbouring conspecifics. In social interactions, individuals can either cooperate - that is behave in a way beneficial to a conspecific - or defect. An individual's fitness depends on the strategies of its interaction partners. Thus, frequency-dependent selection underlies the evolution of cooperation. Apart from interactions between conspecifics, the population composition as a whole can mediate frequency-dependent selection. Here, individuals do not interact with single partners, but "play against the field" (Maynard Smith, 1982). For an example consider coevolving species. Pathogens adapting to common host genotypes can select for rare, resistant host genotypes. Similarly, search image formation in predators can lead to frequency-dependent selection on prey morphs. Pollinator preferences for a common flower morph can exert positive frequency-dependent selection on a plant population.

The evolutionary dynamics of frequency-dependent selection can be explored by evolutionary game theory. In this approach, fitness is determined by the payoff obtained in an evolutionary game, which allows to address very general cases of frequency dependent selection. Traditional evolutionary game theory assumes that population size is infinite, so that stochastic effects can be neglected and deterministic differential equations are obtained. The stable fixed points of the dynamics are the evolutionary stable states (Zeeman, 1980; Taylor and Jonker, 1978; Maynard Smith and Price, 1973; Maynard Smith, 1982; Weibull, 1995; Hofbauer and Sigmund, 1998; Sandholm, 2007).

While some important aspects arising from the finiteness of a population have been realized for a long time (Riley, 1979; Schaffer, 1988; Kandori et al., 1993; Fogel et al., 1998; Ficici and Pollack, 2000; Schreiber, 2001), the concept of weak selection has only recently been transferred from population genetics to evolutionary game theory (Nowak et al., 2004; Taylor et al., 2004). This leads to randomness in birth and death processes. Thus, evolutionary dynamics becomes stochastic.

The traditional concept of evolutionary stable states no longer applies, because neutral drift can lead away from these states. Hence, new definitions of evolutionary stability in finite populations have to be developed (Schaffer, 1988; Fogel et al., 
1998; Nowak et al., 2004; Wild and Taylor, 2004; Lessard and Ladret, 2007; Miekisz, 2008). Instead of fixed points, now the evolutionary dynamics is characterized by the probability of fixation in a particular state and the associated time until fixation (Nowak et al., 2004; Antal and Scheuring, 2006).

Most analytical approaches for evolutionary game dynamics assume that the process that assigns payoffs to individuals is deterministic. This approach is appropriate if an individual's payoff depends only on the composition of the population and not on specific interaction partners. In this case, all individuals using one strategy have the same payoff. Models of this type are good approximations if individuals (e.g. wind-pollinating plant species) interact with all other individuals in the population or with a representative fraction of the population. By contrast, if individuals mate only once or compete with few conspecifics for resources, individuals using the same strategy may have different payoffs, depending on the types of their interaction partners.

There are different ways to incorporate stochasticity into payoff assignment. Fig. 1 shows an overview over some typical scenarios. One possibility is to let individuals have different, stochastic numbers of interactions (Sánchez and Cuesta, 2005; Roca et al., 2006; Traulsen et al., 2007b). Another possibility is to let every individual have only a small number of interactions. In the extreme case, fitness would be determined by only a single interaction. If each individual interacts only with a single other individual drawn randomly from the population, individuals using the same strategy can differ in their payoff-values. This approach is sometimes used in simulations (Doebeli et al., 2004; Hauert et al., 2007, 2008) and often leads to remarkable agreement with analytical calculations in which an individual interacts with all others in the population. In this manuscript we discuss when simulations (based on single interactions) and analytical calculations (based on interactions with all individuals in the population) are expected to give similar results. This leads to the question under which circumstances deterministic versus stochastic payoff assignment results in different evolutionary dynamics. To tackle this question we compare the fixation probabilities for deterministic and stochastic payoff assignment for different evolutionary processes under frequency-dependent selection. 


\section{Evolutionary game dynamics}

We study symmetric two-player games. The two players are assigned payoffs according to the payoff matrix

$$
\begin{aligned}
& \text { A } B \\
& \begin{array}{l}
A \\
B
\end{array}\left(\begin{array}{ll}
a & b \\
c & d
\end{array}\right) \text {. }
\end{aligned}
$$

A player of type $A$ obtains $a$ in interactions with other $A$ players and $b$ in interactions with $B$ players. Equivalently, $B$ players obtain $c$ in interactions with $A$ and $d$ in interactions with $B$. Thus, the payoffs are determined by the players' own phenotypes and the phenotypes of the interaction partners.

In our "All Interaction" scenario (AI) for deterministic payoff assignment, each individual interacts with all other individuals in the population. Thus, the payoff is the average payoff from all interactions. The all interaction scenario is also a good approximation if the number of interactions per individual is so high that the individual payoffs are close to the average payoff. This standard approach is used in the majority of studies of evolutionary game dynamics in unstructured populations. To capture the consequences of stochasticity in payoff assignment, we compare this scenario to the extreme case in which a single interaction determines the reproductive fitness of an individual. In this case (termed SI for single interaction), each individual is randomly assigned an interaction partner. The individual's fitness depends only on this single interaction.

Next, we have to specify the evolutionary process which determines how strategies spread in the population. We consider two different birth-death processes. In the pairwise comparison process, two individuals, a focal individual and a role model, are randomly drawn from the population in each round. The focal individual adopts the strategy of the role model with probability $p$, depending on a payoff comparison. The probability $p$ is given by the Fermi function $p=\left(1+e^{+w\left(\pi_{\text {focal }}-\pi_{\text {role }}\right)}\right)^{-1}$ (Blume, 1993; Szabó and Töke, 1998; Traulsen et al., 2007a; Sandholm, 2007). Here, $w$ determines the intensity of selection. For strong selection, $w \gg 1$, a better 
strategy is always adopted, regardless of the payoff difference. For weak selection, $w \ll 1$, we have $p \approx \frac{1}{2}-w \frac{\pi_{\text {focal }}-\pi_{\text {role }}}{4}$. In this case, the payoffs represent only a small linear disturbance to random strategy adoption.

In the Moran process a single individual reproduces and a randomly selected individual dies in each round (Moran, 1962; Nowak et al., 2004). The probability that a specific individual is chosen for reproduction is proportional to its fitness, which is a function of the payoff obtained in the evolutionary game. Individual fitness $f$ can be evaluated as

(i) a convex combination of background fitness (usually set to one) and payoff $\pi$, $f=1-w+w \pi$ (Nowak et al., 2004) or

(ii) an exponential function $f=e^{+w \pi}$ of payoff $\pi$ (Traulsen et al., 2008).

In both cases, $w$ is the strength of selection and small $w$ corresponds to weak selection.

\section{Stochastic payoff assignment}

\subsection{General remarks}

Let $T^{ \pm}(j)$ denote the probability that the number of $A$ individuals in a population of size $N$ changes from $j$ to $j \pm 1$. If a population consists of $i$ individuals of strategy $A$ the probability of fixation in the all- $A$ state $\phi_{i}$ is given by (Karlin and Taylor, 1975; Nowak, 2006)

$$
\phi_{i}=\frac{1+\sum_{k=1}^{i-1} \prod_{j=1}^{k} \frac{T^{-}(j)}{T^{+}(j)}}{1+\sum_{k=1}^{N-1} \prod_{j=1}^{k} \frac{T^{-}(j)}{T^{+}(j)}} .
$$

Fixation probabilities can only differ between the AI and the SI scenario, if stochasticity in payoff assignment affects the ratio $\gamma(j)=\frac{T^{-}(j)}{T^{+}(j)}$. Formally $\phi_{i}$ is the expectation value of a random variable $X$ that characterizes the evolutionary outcome. Here, $X=1$ if fixation occurs at the all-A state and $X=0$ if fixation occurs at 
the all-B state. Could stochasticity in payoff assignment maintain $\phi_{i}$ but change higher moments of $X$ (e.g. the variance)? In other words, could we miss effects of stochastic payoff assignment if we only analyze $\phi_{i}$ and do not take higher moments into account? Since $X$ is Bernoulli-distributed, all moments of $X$ only depend on $\phi_{i}$ (the variance is e.g. given by $\phi_{i}\left(1-\phi_{i}\right)$ ). Therefore, stochasticity that does not change $\gamma(j)$ can neither change the fixation probability $\phi_{i}$ nor the distribution of $X$.

Thus, we concentrate on stochastic effects that affect the evolutionary outcome by altering $\gamma(j)$. The ratio $\gamma(j)$ determines into which direction the system will move from state $j$ : For $\gamma(j)<1$ the number of $A$ individuals is more likely to increase. For $\gamma(j)>1$ the number of $A$ individuals is more likely to decrease. In the following, we analyze for which parameter values the single interaction scenario changes the $\gamma(j)$ of the all interaction scenario.

\subsection{Pairwise comparison process}

First, we consider the case in which strategies spread according to a pairwise comparison process.

In the simplest scenario each individual interacts with all individuals in the population (abbreviated as AI for all interactions). In this case, $A$ and $B$ individuals have payoffs

$$
\begin{aligned}
\pi_{A} & =\frac{j}{N} a+\frac{N-j}{N} b \\
\pi_{B} & =\frac{j}{N} c+\frac{N-j}{N} d .
\end{aligned}
$$

Note that we have not excluded self-interactions here to keep things as simple as possible. The probability that the number of A individuals increases from $j$ to $j+1$ is $T_{A I}^{+}(j)$. The number of A individuals $j$ can only increase if a B individual compares itself to an A individual. This happens with probability $\frac{j}{N} \frac{N-j}{N-1}$. To obtain $T_{A I}^{+}(j)$ this probability has to be multiplied by the probability that the focal $(B)$ 
individual accepts the role model's strategy $(A)$. The probability $T_{A I}^{-}(j)$ that $\mathrm{j}$ decreases by one is calculated analogously. This yields

$$
T_{A I}^{ \pm}(j)=\frac{j}{N} \frac{N-j}{N-1} \frac{1}{1+e^{\mp w\left(\pi_{A}-\pi_{B}\right)}} .
$$

The ratio of the transition probabilities $\gamma_{A I}(j)$ is then given by

$$
\gamma_{A I}(j)=\frac{T_{A I}^{-}(j)}{T_{A I}^{+}(j)}=e^{-w\left(\pi_{A}-\pi_{B}\right)}=e^{-w\left(\frac{j}{N} a+\frac{N-j}{N} b-\frac{j}{N} c-\frac{N-j}{N} d\right)} .
$$

Now, we compare this result for deterministic payoff assignment with the scenario in which each individual interacts only with a single other individual in each round (abbreviated as SI for single interaction). The probability that a $B$ individual compares itself to an $A$ individual is $\frac{j}{N} \frac{N-j}{N-1}$, the same value as in the AI-scenario discussed above. The acceptance probability depends on the payoffs of the focal individual and the role model. Both partners in the comparison process can interact with $A$ individuals, one can interact with an $A$ individual while the other interacts with a $B$ individual or both partners can interact with $B$ individuals. This leads to the transition probabilities

$$
\begin{aligned}
& T_{S I}^{ \pm}(j)=\frac{N-j}{N} \frac{j}{N-1}\left[\quad\left(\frac{j}{N}\right)^{2} \frac{1}{1+e^{\mp w(a-c)}}+\frac{j}{N} \frac{N-j}{N} \frac{1}{1+e^{\mp w(b-c)}}\right. \\
& \left.+\frac{j}{N} \frac{N-j}{N} \frac{1}{1+e^{\mp w(a-d)}}+\left(\frac{N-j}{N}\right)^{2} \frac{1}{1+e^{\mp w(b-d)}}\right] \text {. }
\end{aligned}
$$

A Taylor expansion for weak selection, $w \ll 1$, leads from Eq. (7) to

$$
T_{S I}^{ \pm}(j)=\frac{N-j}{N} \frac{j}{N-1}\left[\frac{1}{2} \pm w \frac{\pi_{A}-\pi_{B}}{4}\right]
$$

This is identical to the weak selection expansion of $T_{A I}^{ \pm}(j)$, cf. Eq. (5). Again, we denote the ratio of transition probability with $\gamma_{S I}(j)=\frac{T_{S I}^{-}(j)}{T_{S I}^{+}(j)}$. For weak selection, we have $\gamma_{S I}(j)=\gamma_{A I}(j)$ for all $j$. Thus, the fixation probabilities in the scenario with stochastic payoff assignment are the same as in the scenario with deterministic payoff assignment (compare Fig. 2).

If selection is strong, however, the exponential function cannot be linearized. Then, $\gamma_{S I}(j)=\gamma_{A I}(j)$ only holds, if $a=b$ and $c=d$. But in this special case, payoffs do 
not depend on interactions at all and thus, selection is frequency independent. For all other payoff values, i.e. whenever selection is frequency dependent, the fixation probabilities in the SI scenario can differ from those obtained with the AI scenario. For example, in coordination games with $a>c$ and $b<d$, this can change the point $j^{*}$ where the direction of selection changes, i.e. the solution of $T^{+}(j)=T^{-}(j)$. For the AI scenario we obtain analytically $j_{A I}^{*}=(d-b) N /(a-b-c+d)$ for all intensities of selection. In the SI scenario $j^{*}$ depends on the intensity of selection and can be determined numerically. While $j_{S I}^{*} \approx j_{A I}^{*}$ for small $w$, higher intensities of selection can lead to differences (compare Fig. 2). For $w \rightarrow \infty$, only the payoff ranking is of importance, as one can infer from the transition probabilities under strong selection, Eq. (7). For example, for payoff matrices with $a>d>c>b$, we have $j_{S I}^{*} \rightarrow N / 2$. For payoff matrices with $a>d>b>c$, we have $j_{S I}^{*} \rightarrow$ $(1-1 / \sqrt{2}) N \approx 0.293 N$.

To explore in more detail how the choice of the interaction scenario affects the fixation probabilities, we analyze the ratio $R(j)$ of the transition probabilities in the SI scenario and the transition probabilities in the AI scenario.

$$
R(j)=\frac{\gamma_{S I}(j)}{\gamma_{A I}(j)}>1 \quad \text { for } \quad 0<j<N \Rightarrow B \text { favoured in single interaction }
$$

From the general form of $R(j)$, it is not obvious whether $R(j)>1$ or $R(j)<1$.

For frequency independent selection, $a=b$ and $c=d$, there is no difference between the scenarios, leading to $R(j)=1$. For neutral selection, $w=0$, we have $R(j)=1$ for all $j$. Next, we study general payoff matrices under weak selection by expanding $R(j)$ in a Taylor Series.

$$
\begin{aligned}
R(j) \approx 1 & +\frac{j(N-j)}{12 N^{3}}\left[j(a-b-c+d)^{3}\right. \\
& +N\left(a^{3}+2 b^{3}-c^{3}-2 d^{3}\right) \\
& \left.+3 N\left(d^{2}(b+c)-b^{2}(a+d)+2 b d(a-c)+b c^{2}-a^{2} d\right)\right] w^{3}
\end{aligned}
$$


Interestingly, both the linear and the quadratic terms of the Taylor expansion in $w$ are zero. Thus, the cubic term determines whether $R(j)$ is larger or smaller than one for weak selection. This implies that selection does not have to be extremely weak to make the difference between AI and SI vanish. As a concrete example, let us consider the special case in which the fitness of strategy $A$, is constant, $a=b$. For $3 a>c+2 d$, the $R(j)>1$ for all $j$, so that $B$ is favored in the single interaction scenario compared to the all interaction scenario. Interestingly, this is equivalent to $\frac{d-b}{a-b-c+d}<\frac{1}{3}$, i.e. the unstable equilibrium of a coordination game has to be closer to $B$ than $\frac{1}{3}$ for $B$ to be favored in the single interaction scenario compared to the all interaction scenario, which reminds of the 1/3-rule (Nowak et al., 2004; Ohtsuki et al., 2007).

In the above equations, pairs for payoff assignment are formed by drawing individuals with replacement. This means that both the focal individual and the role model could interact with the same individual. Moreover, we allowed self-interactions in order to keep the calculations as transparent as possible. In Appendix A, we derive expressions for the ratios of the transition probabilities that take into account that each individual interacts with exactly one other individual. Our main conclusions also hold in this more sophisticated scenario: If selection is weak, $\gamma_{S I}(j)=\gamma_{A I}(j)$ for all $j$ and the fixation probabilities are identical. For strong selection, however, we have in general $\gamma_{S I}(j) \neq \gamma_{A I}(j)$ and the two scenarios will lead to different fixation probabilities.

\subsection{Moran process}

In the Moran process, $T^{+}(j)$ is the probability to choose an $A$ individual proportional to fitness for reproduction and to choose a $B$ individual at random for death. The probability that an $A$ individual is chosen for reproduction is given by the sum of all fitness values of $A$ individuals divided by the sum of the fitness values of all individuals in the population. Since the probability that a B individual dies is $\frac{N-j}{N}$, 
we obtain

$$
T^{+}(j)=\frac{\sum f_{A}}{\sum f_{A}+\sum f_{B}} \frac{N-j}{N} .
$$

Here, $\sum f_{A}$ is the sum of all fitness values of $A$ individuals and $\sum f_{B}$ is the sum of all fitness values of $B$ 's.

If each individual interacts with all other individuals and fitness is a linear function of payoff, the all interaction scenario leads to

$$
\begin{aligned}
& \sum f_{A}=j\left(1-w+w\left[\frac{j-1}{N-1} a+\frac{N-j}{N-1} b\right]\right) \\
& \sum f_{B}=(N-j)\left(1-w+w\left[\frac{j}{N-1} c+\frac{N-j-1}{N-1} d\right]\right) .
\end{aligned}
$$

For the transition probabilities, we obtain

$$
\begin{aligned}
& T_{A I}^{+}=\frac{j\left(1-w+w\left[\frac{j-1}{N-1} a+\frac{N-j}{N-1} b\right]\right)}{N(1-w)+w\left[j\left(\frac{j-1}{N-1} a+\frac{N-j}{N-1} b\right)+(N-j)\left(\frac{j}{N-1} c+\frac{N-j-1}{N-1} d\right)\right]} \frac{N-j}{N} \\
& T_{A I}^{-}=\frac{(N-j)\left(1-w+w\left[\frac{j}{N-1} c+\frac{N-j-1}{N-1} d\right]\right)}{N(1-w)+w\left[j\left(\frac{j-1}{N-1} a+\frac{N-j}{N-1} b\right)+(N-j)\left(\frac{j}{N-1} c+\frac{N-j-1}{N-1} d\right)\right]} \frac{j}{N} .
\end{aligned}
$$

Thus, the ratio of the transition probabilities in the all interaction scenario is

$$
\gamma_{A I}(j)=\frac{T_{A I}^{-}(j)}{T_{A I}^{+}(j)}=\frac{1-w+w\left[\frac{j}{N-1} c+\frac{N-j-1}{N-1} d\right]}{1-w+w\left[\frac{j-1}{N-1} a+\frac{N-j}{N-1} b\right]} .
$$

In the single interaction scenario an $A$ individual can have fitness $f_{A A}=1-w+w a$, if it interacts with another $A$, or $f_{A B}=1-w+w b$, if it interacts with $B$. Similarly, a $B$ individual can have fitness $f_{B A}=1-w+w c$ or $f_{B B}=1-w+w d$. In this scenario $\sum f_{A}$ and $\sum f_{A}+\sum f_{B}$ are random variables. Since these two random variables are not independent of each other, the ratio of the expected values does not give the expected value for the ratio, i.e. $T_{S I}^{+}(j)$. To derive an expression for $T_{S I}^{+}(j)$, we use the assumption that each individual interacts with exactly one other individual per round. A similar scenario was discussed by Miekisz (2005). 
Thus, $N$ must be even. Consider the case where also $j$ is even. It follows that the number of mixed pairs can only be even. We calculate the probability that exactly $2 k$ mixed pairs (an $A$ and a $B$ individual interact with each other) are formed. There are $\left(\begin{array}{c}N \\ j\end{array}\right)$ possibilities to arrange the $j$ type $A$ individuals among the $N$ individuals. The number of possibilities to arrange the $2 k$ mixed pairs among the $\frac{N}{2}$ pair positions is $\left(\begin{array}{c}\frac{N}{2} \\ 2 k\end{array}\right)$. Since each mixed pair can be written in two ways $(A B$ and $B A)$, we multiply by $2^{2 k}$. The remaining $j-2 k$ type $A$ individuals form $\frac{j-2 k}{2} A A$ pairs. The number of possibilities to arrange these AA pairs among the remaining $\frac{N}{2}-2 k$ pair positions is $\left(\begin{array}{c}\frac{N}{2}-2 k \\ \frac{j}{2}-k\end{array}\right)$. Hence, we arrive at

$$
\begin{aligned}
T_{S I}^{+}(j) & =\frac{1}{\left(\begin{array}{c}
N \\
j
\end{array}\right)} \sum_{k=0}^{j / 2}\left(\begin{array}{c}
\frac{N}{2} \\
2 k
\end{array}\right) 2^{2 k}\left(\begin{array}{c}
\frac{N}{2}-2 k \\
\frac{j}{2}-k
\end{array}\right) \\
& \times \frac{(j-2 k) f_{A A}+2 k f_{A B}}{(j-2 k) f_{A A}+2 k f_{A B}+2 k f_{B A}+(N-j-2 k) f_{B B}} \frac{N-j}{N}
\end{aligned}
$$

$T_{S I}^{-}(j)$ and equations for uneven $j$ can be calculated analogously (see Appendix B). In general, we find $T_{A I}^{ \pm}(j) \neq T_{S I}^{ \pm}(j)$. The single interaction scenario does have an effect in the Moran process with linear payoff to fitness assignment, because this process depends not only on the fitness of a focal individual, but also takes the background fitness into account. Again, for weak selection, $w \ll 1$, the transition probabilities of the two scenarios lead to the same dynamics (compare Fig. $3)$. Thus, the effect of the single interaction scenario is only important for strong selection. The transition probabilities are also identical if selection becomes frequency independent, $a=b$ and $c=d$, because payoffs become independent of the interaction partner in this case.

Fluctuations around the expected fraction of mixed pairs decrease with increasing population size $N$. The expected $k$ is given by

$$
k_{\text {expected }}(j)=\frac{1}{\left(\begin{array}{c}
N \\
j
\end{array}\right)} \sum_{k=0}^{j / 2}\left(\begin{array}{c}
\frac{N}{2} \\
2 k
\end{array}\right) 2^{2 k}\left(\begin{array}{c}
\frac{N}{2}-2 k \\
\frac{j}{2}-k
\end{array}\right) k=\frac{j}{2} \frac{N-j}{N-1}
$$

The expected fraction of mixed pairs is thus

$$
\frac{2 k_{\text {expected }}(j)}{\frac{N}{2}}=\frac{2 j}{N} \frac{N-j}{N-1}
$$


If we replace the average over $k$ in Eq. (17) by $k_{\text {expected }}(j)$, we obtain $T_{S I}^{+}(j)=\frac{j\left(f_{A A}(j-1)+f_{A B}(N-j)\right)}{j\left(f_{A A}(j-1)+f_{A B}(N-j)\right)+(N-j)\left(f_{B A} j+f_{B B}(N-j-1)\right)} \frac{N-j}{N}$.

This is equal to $T_{A I}^{+}(j)$. In large populations where stochasticity of pairing has no effect, the transition probabilities (and thus the fixation probabilities) of the two scenarios are identical if fitness is a linear function of payoff. However, if fitness is an exponential function of payoff, the single interaction scenario leads to higher average fitness values. This can be shown by comparing the average fitness of an individual in the AI scenario $f^{A I}(x)=e^{x \pi_{1}+(1-x) \pi_{2}}$ to the average fitness of an individual in the SI scenario $f^{S I}(x)=x e^{\pi_{1}}+(1-x) e^{\pi_{2}}$. Here, $x=\frac{j}{N}$ is the proportion of $A$ individuals and $\pi_{1}$ and $\pi_{2}$ are the individual's payoffs upon interaction with an $\mathrm{A}$ or $\mathrm{B}$ individual respectively. The linear function $f^{S I}(x)$ and the exponential function $f^{A I}(x)$ intersect at exactly two points: $x_{0}=0$ and $x_{1}=1$. Since the exponential function has positive curvature, $f^{A I}(x)<f^{S I}(x)$ for $0<$ $x<1$.

To explore which strategy is favoured in the single interaction scenario in large populations where the stochasticity of pairing is negligible, we analyze $R(x)$ (the ratio of the transition probabilities in the SI scenario and the transition probabilities in the AI scenario).

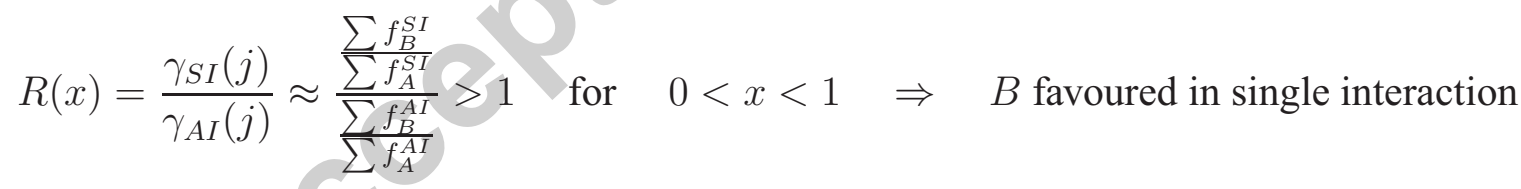

For the exponential payoff to fitness mapping we find for $R(x)$

$$
R(x)=e^{\frac{N}{N-1} w\left(x(a-b-c+d)+\frac{d-a}{N}+b-d\right)} \frac{x e^{w c}+\left(1-x-\frac{1}{N}\right) e^{w d}}{\left(x-\frac{1}{N}\right) e^{w a}+(1-x) e^{w b}} .
$$

The $\frac{1}{N}$ corrections only arise because we explicitly excluded self-interactions. The population composition $x$ and the payoff values determine if $R(x)>1$ or $R(x)<$ 1 . For large $N$, we have $R(0)=R(1)=1$. Only in special cases, $R(x)$ will be larger or smaller than one for all values of $x$. Consider the special case $a=b$, in which strategy $A$ always obtains the same payoff, regardless of $x$. The derivative $\frac{d R(x)}{d x}$ has only a single root, which means that $R(x)$ either has a maximum or 
a minimum. Moreover, $\left.\frac{d R(x)}{d x}\right|_{x=0}>0$, such that $R(x) \geq 1$ for $0 \leq x \leq 1$. Thus, strategy $\mathrm{B}$ has a higher fixation probability in the single interaction compared to the all interactions scenario for $a=b$. In other words, a strategy with frequency dependent fitness is always favored by the SI scenario, whereas the strategy with constant fitness is favored by the AI scenario. This is a stark contrast to the model based on pairwise comparison discussed above. In the general case when both fitness values are frequency dependent, it is more difficult to determine which strategy is favored by the SI scenario.

\section{Conclusion}

Our results show that selection intensity determines if the evolutionary dynamics of the all interaction scenario and that of the single interaction scenario differ. While the fixation probabilities in the two scenarios become similar if selection is weak, strong selection generally leads to differences. This finding holds for the pairwise comparison process typically modelling cultural spreading of strategies and the Moran process usually modelling Darwinian evolution.

Especially in economics, evolutionary game dynamics is typically treated as deterministic and selection is strong. In this case, it can make a significant difference if the payoff itself is determined by a single (or a few) interactions. The stochasticity in the payoffs can render the whole dynamics stochastic, even if the intensity of selection is very large.

How can we understand intuitively that the choice of the payoff assignment scenario has an effect only if selection is strong? The expected payoff of each individual is the same in the single and all interaction scenario. In the Moran process the function mapping payoff to fitness can be approximated by a linear function if selection is weak. In this case the expected fitness of each individual and hence the fixation probabilities are identical in the two scenarios. Strong selection requires a nonlinear mapping function that ensures that even small differences in payoff have strong effects on fitness. Only in this case, the direction of the evolutionary dy- 
namics becomes deterministic. As the mapping function is nonlinear, the expected fitness of an individual depends on the payoff distribution. As a result the fixation probabilities differ between the scenarios with stochastic versus deterministic payoff assignment.

For the pairwise-comparison process an analogous reasoning applies. The expected payoff difference between a randomly drawn $A$ and a randomly drawn $B$ individual is the same for the two scenarios. If selection is weak, transition probabilities are a linear function of the payoff difference. By contrast, strong selection, which is equivalent to nonlinear mapping, leads to different evolutionary outcomes for stochastic versus deterministic payoff assignment.

To summarize, we have shown that for weak selection, the interaction scenario (cf. Fig. 1) has a small influence on the system. But for strong selection, the choice of the interaction scenario can change the resulting evolutionary dynamics.

\section{A Interactions with exactly one other individual in the pairwise comparison process}

Here, we derive 'exact' (that is, excluding self-interactions) expressions for the transition probabilities in the pairwise comparison process with linear payoff to fitness mapping. First, we consider the single interaction scenario. Since a focal $B$ individual with payoff $c$ or $d$ can compare itself to a role model of type $A$, which can either have payoff $a$ or $b$, we have to consider four cases that can lead to an increase in the number of $\mathrm{A}$ individuals.

We only discuss one of them in detail here, because the remaining three cases follow from analogous arguments. A $B$ individual with payoff $c$ as focal individual is paired with an $A$ individual with payoff $a$ as a role model: With probability $\frac{N-j}{N}$ a $B$ individual is chosen as focal individual. This $B$ individual interacts with an $A$ individual and thus obtains payoff $c$ with probability $\frac{j}{N-1}$. The probability that the role model is not the focal individual's interaction partner is $\frac{N-2}{N-1}$. From the remain- 
ing individuals an $A$ individual is drawn as a role model with probability $\frac{j-1}{N-2}$. The probability that the role model interacts with an $A$ individual and obtains payoff $a$ is $\frac{j-2}{N-3}$. The probabilities for the three other cases can be calculated analogously. The transition probability $T_{S I}^{+}(j)$ is then given by

$$
\begin{aligned}
T_{S I}^{+}(j) & =\frac{N-j}{N} \frac{j}{N-1} \frac{N-2}{N-1} \frac{j-1}{N-2} \frac{j-2}{N-3} \frac{1}{1+e^{w(c-a)}} \\
& +\frac{N-j}{N} \frac{j}{N-1}\left(\frac{1}{N-1}+\frac{N-2}{N-1} \frac{j-1}{N-2} \frac{N-i-1}{N-3}\right) \frac{1}{1+e^{w(c-b)}} \\
& +\frac{N-j}{N-j-1} \frac{N-2}{N-1} \frac{j}{N-1} \frac{j-1}{N-3} \frac{1}{1+e^{w(d-a)}} \\
& +\frac{N-j}{N} \frac{N-j-1}{N-1} \frac{N-2}{N-1} \frac{j}{N-2} \frac{N-j-2}{N-3} \frac{1}{1+e^{w(d-b)}} \\
& =\frac{j(N-j)}{N(N-3)(N-1)^{2}}\left[\frac{(j-2)(j-1)}{1+e^{w(c-a)}+\frac{j(N-j)-2}{1+e^{w(c-b)}}}\right. \\
& \left.+\frac{(j-1)(N-j-1)}{1+e^{w(d-a)}}+\frac{(N-j-1)(N-j-2)}{1+e^{w(d-b)}}\right]
\end{aligned}
$$

The probability $T_{S I}^{-}(j)$ can be calculated in the same way. Next, we calculate the corresponding transition probabilities for the AI scenario. We obtain

$$
T_{A I}^{ \pm}(j)=\frac{j}{N} \frac{N-j}{N-1} \frac{1}{1+e^{\mp w\left(\frac{j-1}{N-1} a+\frac{N-j}{N-1} b-\frac{j}{N-1} c-\frac{N-j-1}{N-1} d\right)}},
$$

which is significantly simpler than Eq. (A.1).

\section{B Single interaction transition probabilities in the Moran process}

Here, we present the transition probabilities in the scenario in which payoff assignment is based on a single interaction and strategies spread according to a Moran process. If the number of $A$ individuals $j$ is even, we have

$$
\begin{aligned}
T_{S I}^{+}(j) & =\frac{1}{\left(\begin{array}{c}
N \\
j
\end{array}\right)} \sum_{k=0}^{j / 2}\left(\begin{array}{c}
\frac{N}{2} \\
2 k
\end{array}\right) 2^{2 k}\left(\begin{array}{c}
\frac{N}{2}-2 k \\
\frac{j}{2}-k
\end{array}\right) \\
& \times \frac{(j-2 k) f_{A A}+2 k f_{A B}}{F(2 k)} \frac{N-j}{N}
\end{aligned}
$$




$$
\begin{aligned}
T_{S I}^{-}(j) & =\frac{1}{\left(\begin{array}{c}
N \\
j
\end{array}\right)} \sum_{k=0}^{j / 2}\left(\begin{array}{c}
\frac{N}{2} \\
2 k
\end{array}\right) 2^{2 k}\left(\begin{array}{c}
\frac{N}{2}-2 k \\
\frac{j}{2}-k
\end{array}\right) \\
& \times \frac{(N-j-2 k) f_{B B}+2 k f_{B A}}{F(2 k)} \frac{j}{N} .
\end{aligned}
$$

The total fitness in a system with $l$ interactions between $A$ and $B$ is given by $F(l)=$ $(j-l) f_{A A}+l f_{A B}+l f_{B A}+(N-j-l) f_{B B}$. For odd $j$, the transition probabilities are given by

$$
\begin{aligned}
T_{S I}^{+}(j) & =\frac{1}{\left(\begin{array}{c}
N \\
j
\end{array}\right)} \sum_{k=0}^{(j-1) / 2}\left(\begin{array}{c}
\frac{N}{2} \\
2 k+1
\end{array}\right) 2^{2 k+1}\left(\begin{array}{c}
\frac{N}{2}-(2 k+1) \\
\frac{j-(2 k+1)}{2}
\end{array}\right) \\
& \times \frac{(j-1-2 k) f_{A A}+(2 k+1) f_{A B} \frac{N-j}{N}}{F(2 k+1)} \\
T_{S I}^{-}(j) & =\frac{1}{\left(\begin{array}{c}
N \\
j
\end{array}\right)} \sum_{k=0}^{(j-1) / 2}\left(\begin{array}{c}
\frac{N}{2} \\
2 k+1
\end{array}\right) 2^{2 k+1}\left(\begin{array}{c}
\frac{N}{2}-(2 k+1) \\
\frac{j-(2 k+1)}{2}
\end{array}\right) \\
& \times \frac{(N-j-1-2 k) f_{B B}+(2 k+1) f_{B A}}{F(2 k+1)} .
\end{aligned}
$$

\section{References}

Antal, T., Scheuring, I., 2006. Fixation of strategies for an evolutionary game in finite populations. Bull. Math. Biol. 68, 1923-1944.

Blume, L. E., 1993. The statistical mechanics of strategic interaction. Games and Economic Behavior 4, 387-424.

Doebeli, M., Hauert, C., Killingback, T., Oct 2004. The evolutionary origin of cooperators and defectors. Science 306 (5697), 859-62.

Ficici, S., Pollack, J., 2000. Effects of finite populations on evolutionary stable strategies. In: Whitley, D., Goldberg, D., Cantu-Paz, E., Spector, L., Parmee, I., Beyer, H.-G. (Eds.), Proceedings GECCO. Morgan-Kaufmann, San Francisco, pp. 927-934.

Fogel, G., Andrews, P., Fogel, D., 1998. On the instability of evolutionary stable strategies in small populations. Ecol. Model. 109, 283-294.

Hauert, C., Traulsen, A., Brandt, H., Nowak, M. A., Sigmund, K., 2007. Via freedom to coercion: the emergence of costly punishment. Science 316, 1905-1907. 
Hauert, C., Traulsen, A., Brandt, H., Nowak, M. A., Sigmund, K., 2008. Public goods with punishment and abstaining in finite and infinite populations. Biological Theory 3, 114-122.

Hofbauer, J., Sigmund, K., 1998. Evolutionary Games and Population Dynamics. Cambridge University Press, Cambridge.

Kandori, M., Mailath, G. J., Rob, R., 1993. Learning, mutation, and long run equilibria in games. Econometrica 61, 29-56.

Karlin, S., Taylor, H. M. A., 1975. A first course in stochastic processes, 2nd Edition. Academic, London.

Lessard, S., Ladret, V., 2007. The probability of fixation of a single mutant in an exchangeable selection model. J. Math. Biol. 54, 721-744.

Maynard Smith, J., 1982. Evolution and the Theory of Games. Cambridge University Press, Cambridge.

Maynard Smith, J., Price, G. R., 1973. The logic of animal conflict. Nature 246, 15-18.

Miekisz, J., 2005. Equilibrium selection in evolutionary games with random matching of players. J. Theor. Biol. 232, 47-53.

Miekisz, J., 2008. Evolutionary game theory and population dynamics. Lecture Notes In Mathematics 1940, 269-316.

Moran, P. A. P., 1962. The statistical processes of evolutionary theory. Clarendon, Oxford.

Nowak, M. A., 2006. Evolutionary Dynamics. Harvard University Press, Cambridge, MA.

Nowak, M. A., Sasaki, A., Taylor, C., Fudenberg, D., 2004. Emergence of cooperation and evolutionary stability in finite populations. Nature 428, 646-650.

Ohtsuki, H., Bordalo, P., Nowak, M. A., 2007. The one-third law of evolutionary dynamics. Jour. Theor. Biol. 249, 289-295.

Riley, J. G., 1979. Evolutionary equilibrium strategies. J. Theor. Biol. 76, 109-123.

Roca, C. P., Cuesta, J. A., Sánchez, A., 2006. Time scales in evolutionary dynamics. Phys. Rev. Letters 97, 158701.

Sánchez, A., Cuesta, J. A., 2005. Altruism may arise from individual selection. Jour. Theor. Biol. 235, 233-240. 
Sandholm, W. H., 2007. Population games and evolutionary dynamics. MIT Press, Cambridge, MA.

Schaffer, M., 1988. Evolutionary stable strategies for a finite population and variable contest size. J. Theo. Biol. 132, 469-478.

Schreiber, S., 2001. Urn models, replicator processes, and random genetic drift. Siam J. Appl. Math. 61, 2148-2167.

Szabó, G., Fáth, G., 2007. Evolutionary games on graphs. Physics Reports 446, 97-216.

Szabó, G., Töke, C., 1998. Evolutionary Prisoner's Dilemma game on a square lattice. Phys. Rev. E 58, 69.

Taylor, C., Fudenberg, D., Sasaki, A., Nowak, M. A., 2004. Evolutionary game dynamics in finite populations. Bull. Math. Biol. 66, 1621-1644.

Taylor, P. D., Jonker, L., 1978. Evolutionary stable strategies and game dynamics. Math. Biosci. 40, 145-156.

Traulsen, A., Pacheco, J. M., Nowak, M. A., 2007a. Pairwise comparison and selection temperature in evolutionary game dynamics. J. Theor. Biol. 246, 522-529.

Traulsen, A., Pacheco, J. M., Nowak, M. A., 2007b. Stochastic payoff evaluation increases the temperature of selection. J. Theor. Biol. 244, 349-357.

Traulsen, A., Shoresh, N., Nowak, M. A., 2008. Analytical results for individual and group selection of any intensity. Bull. Math. Biol. 63, 363.

Weibull, J., 1995. Evolutionary Game Theory. MIT Press, Cambridge.

Wild, G., Taylor, P., 2004. Fitness and evolutionary stability in game theoretic models of finite populations. Proc. Roy. Soc. Lond. B 271, 2345-2349.

Zeeman, E. C., 1980. Population dynamics from game theory. Lecture Notes in Mathematics, 819.

\section{Caption Fig.1:}

Four examples for interaction scenarios in a population of $N=20$. In contrast to games on fixed networks (Szabó and Fáth, 2007), interaction partners change in each time step. (a) In the all interaction scenario, every individual interacts with all other $N-1$ individuals. The payoffs thus only depend on the type of the individual. (b) If every individual has a large number of interactions $L \gg 1$ (shown for 
$\mathrm{L}=5$ ), each individual will interact with a representative fraction of the other types. Consequently, the payoffs depend only on the type of the individual, as in the all interaction scenario. (c) If every interaction occurs with probability p (shown for $p=0.1$ ), then some individuals may have few or no interactions and some may have many, leading to stochastic payoffs. This stochastic interaction scenario has been addressed in (Traulsen et al., 2007). (d) If every individual has exactly one interaction, each type can obtain two different payoff values that are obtained with a probability given by the frequency of the two types. Since (a) and (d) are the most extreme cases, we concentrate on the comparison of these two in the present paper.

\section{Caption Fig.2:}

Fixation probability of $A$ individuals in the pairwise comparison process for a coordination game (both strategies are best replies to themselves) in a population of size $N=100$. Lines show the analytical results and symbols show results from numerical simulations. Both agree perfectly with each other. For weak selection $(w=0.1)$ the all interaction scenario and the single interaction scenario coincide, as predicted. For strong selection $(w=3.0)$ the choice of the interaction scenario strongly affects fixation probabilities. In the all interaction scenario, the direction of selection changes at $j_{A I}^{*}=40$ for all intensities of selection. In the single interaction scenario the point where the direction of selection changes, depends on selection intensity: We find numerically $j_{S I}^{*} \approx 40$ for low selection intensity $(w=0.1)$, and $j_{S I}^{*} \approx 49$ for $w=3.0$.

\section{Caption Fig.3:}

Fixation probability of $A$ individuals in the Moran process with exponential payoff to fitness mapping for a coordination game in a population of size $N=100$. Analytical results (lines) and simulations (symbols) agree perfectly with each other. For weak selection $(w=0.1)$ the all interaction scenario and the single interaction scenario coincide, as predicted. For strong selection $(w=3.0)$ the choice of the interaction scenario changes the fixation probabilities. The all interaction scenario is identical to the pairwise comparison process, since the ratio of the transition probabilities is identical. Only in the single interaction scenario, the root 
of $g(j)=T^{+}(j)-T^{-}(j)$, where the direction of selection changes, depends on the intensity of selection $w$. For $w=0.1$, we find numerically $j_{S I}^{*} \approx 37$ and for $w=3.0 j_{S I}^{*} \approx 3$. For $a>b, c, d$ and $w \rightarrow \infty$, we have $T_{S I}^{-}(j) \rightarrow 0$ for $j>1$ and $T_{S I}^{+}(j) \rightarrow \frac{N-j}{N}$ (compare Eq. (20)). Thus, if selection is sufficiently strong, $T_{S I}^{+}(j)>T_{S I}^{-}(j)$ for $1<j<N$, so that $\phi_{j} \rightarrow 1$ for $j>1$, in contrast to the pairwise comparison process, see Fig. 2. 
(a)

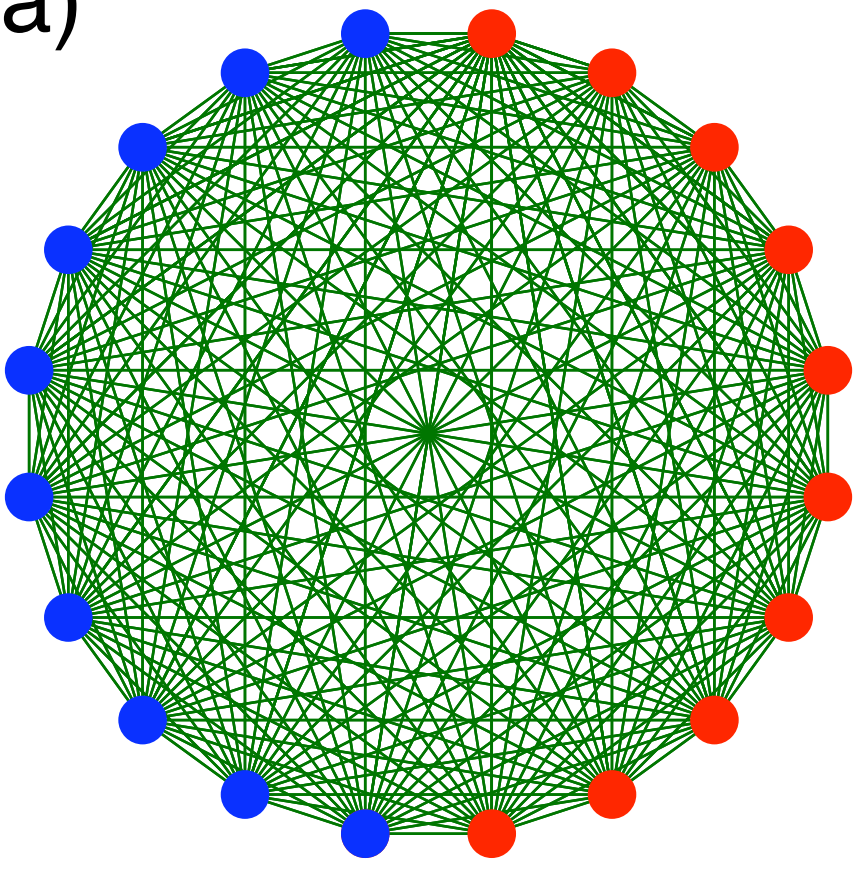

All Interaction

(c)

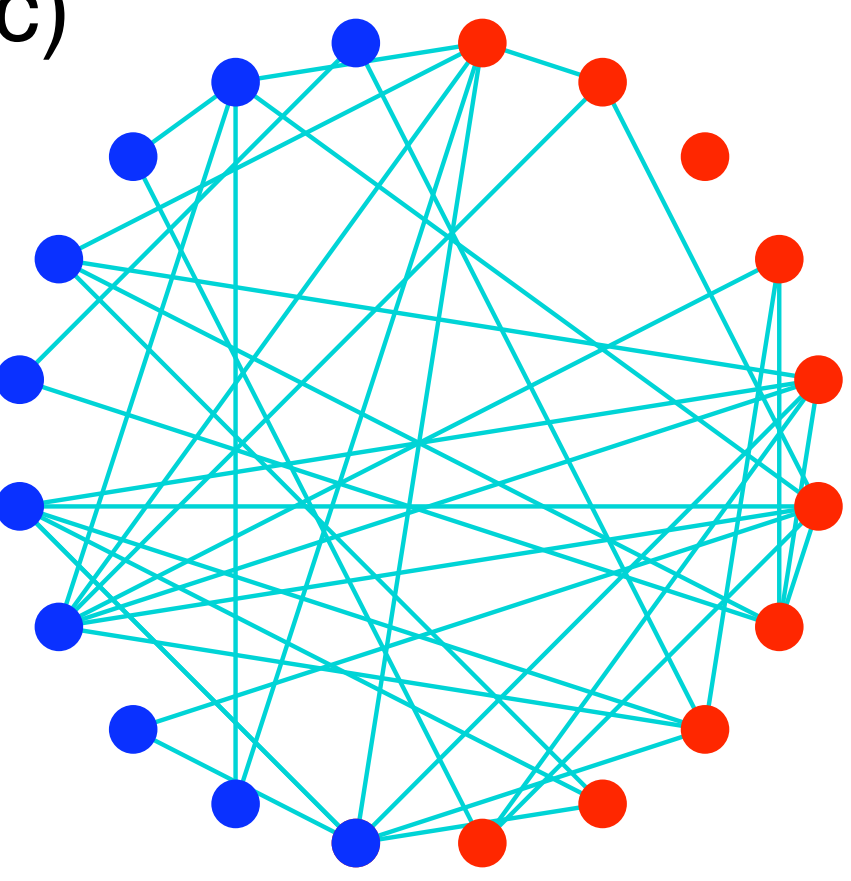

Stochastic Interaction (b)

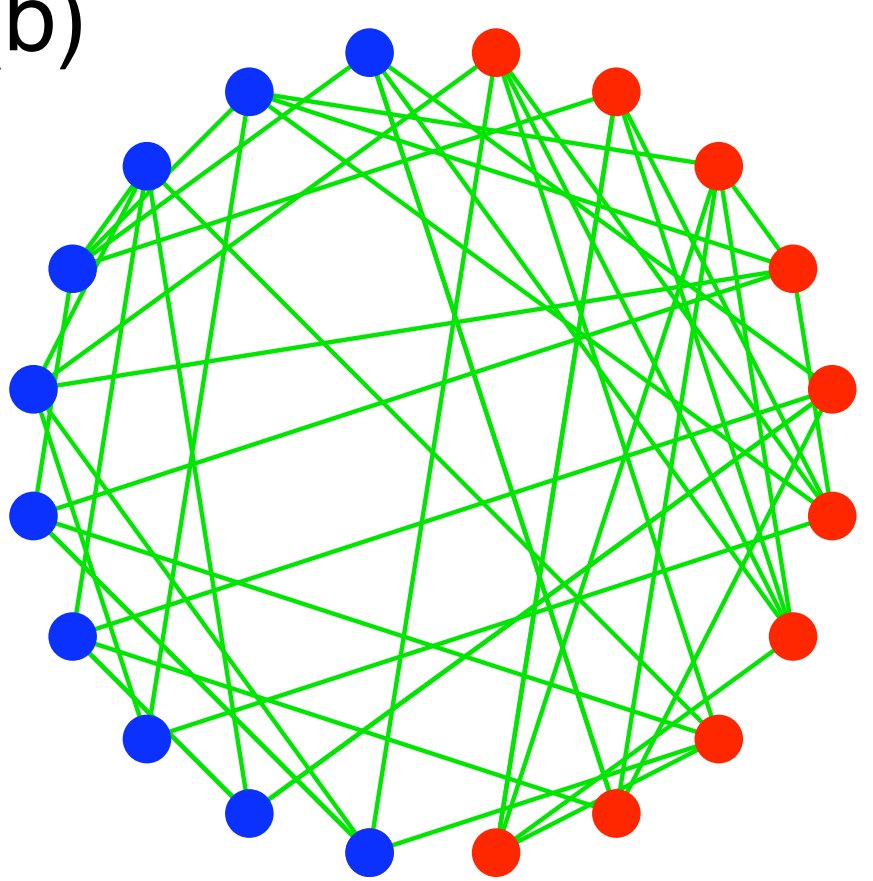

Representative

Interaction

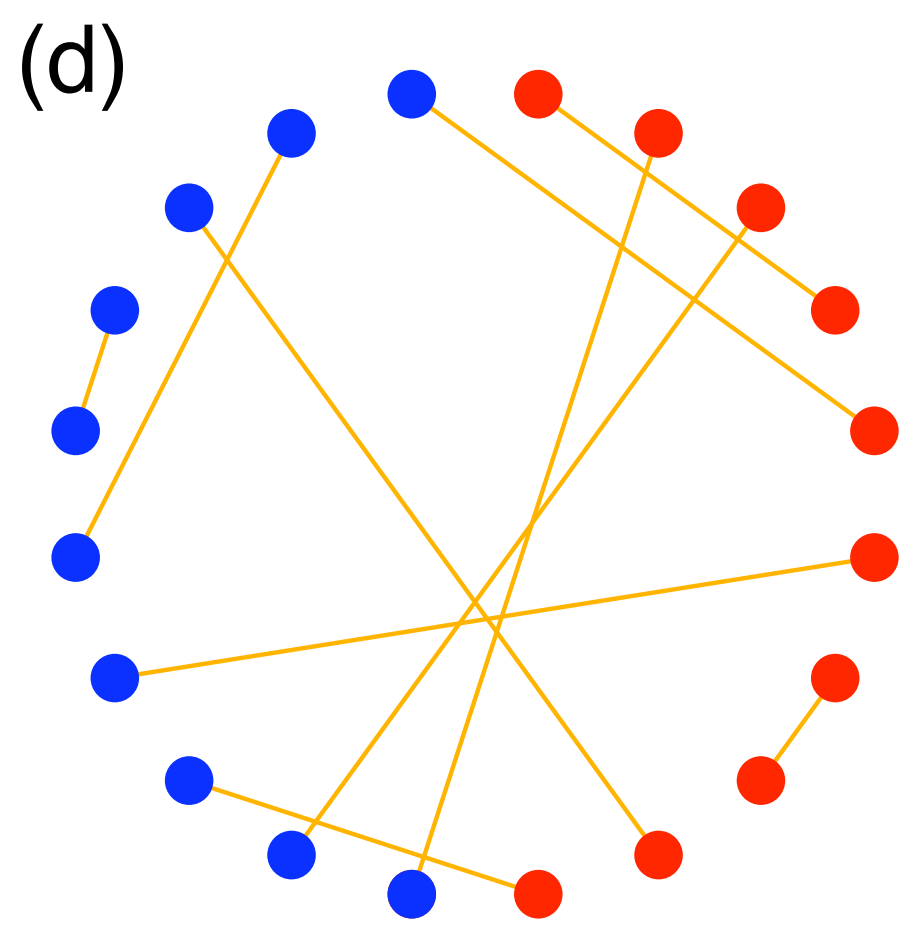

Single Interaction 


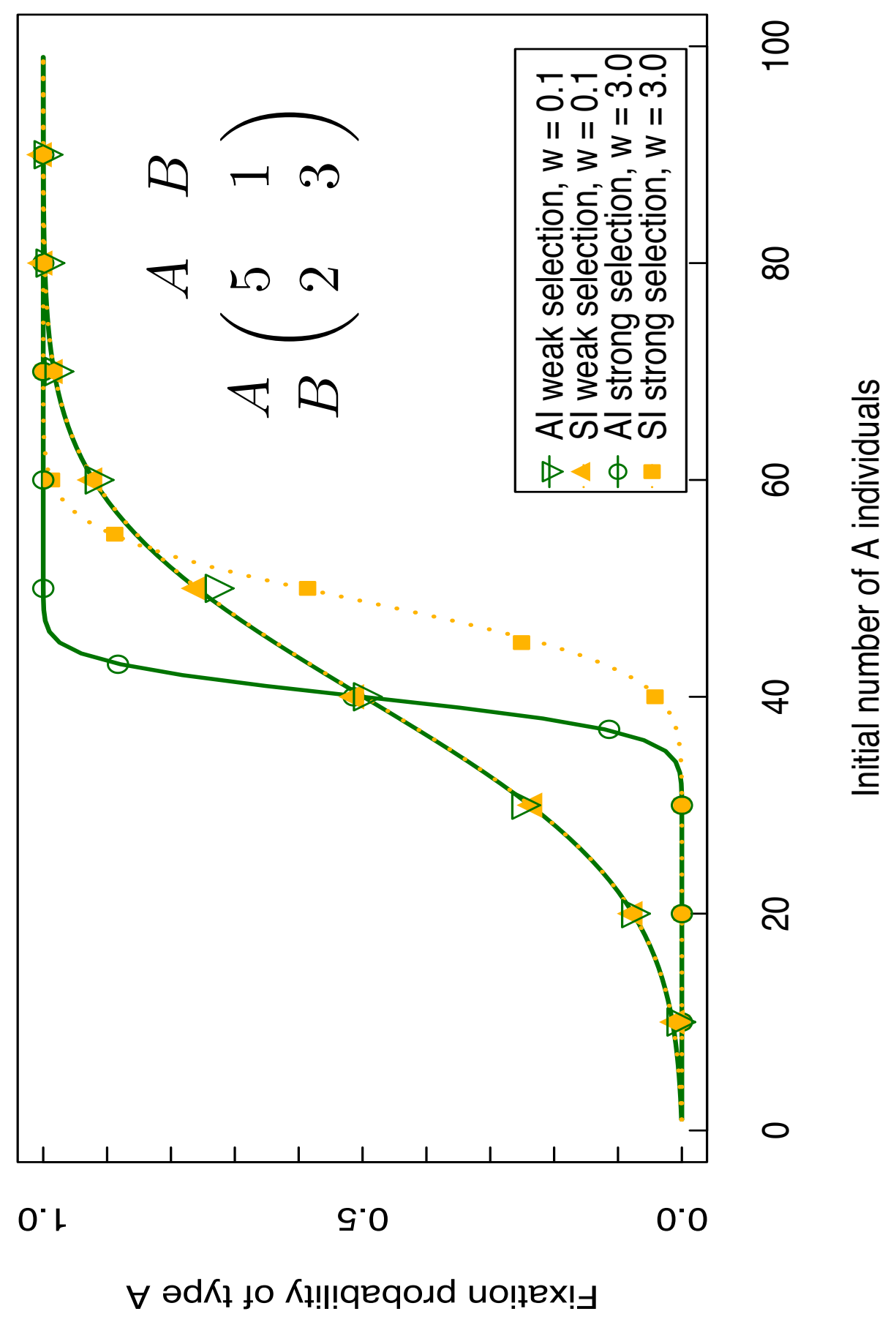




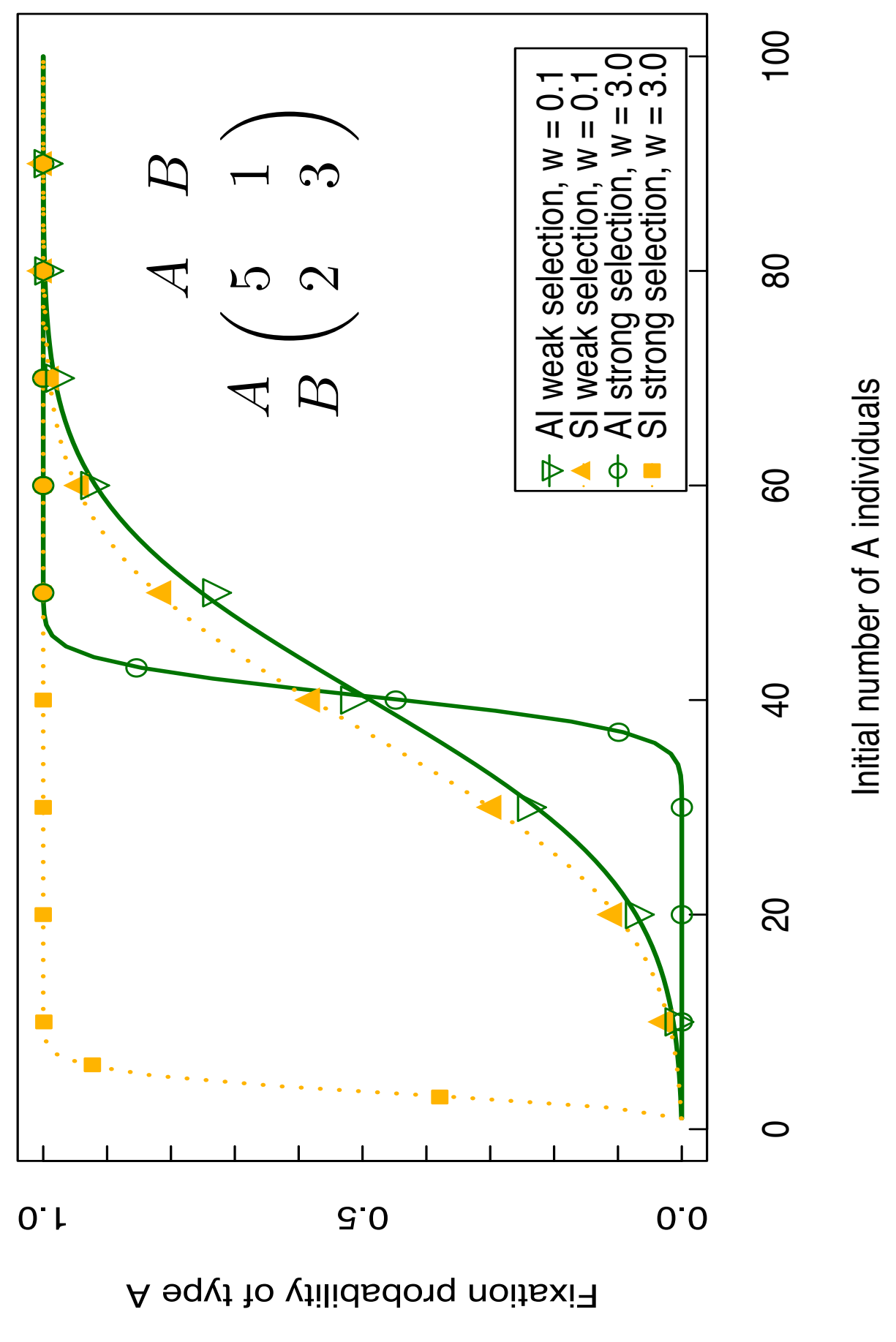

